\title{
THE METHOD OF UPPER AND LOWER SOLUTIONS FOR SOME NONLINEAR BOUNDARY VALUE PROBLEMS IN UNBOUNDED DOMAINS
}

\author{
NGUYÊN PHUONG CÁC
}

1. Introduction. Let $D$ be a bounded domain in the Euclidean space $\mathbf{R}^{N}(N \geqq 2)$ and let $G=\mathbf{R}^{N}-\bar{D}$ where $\bar{D}$ is the closure of $D$. We assume that the boundary $\partial G$ of $G$ is smooth. Consider the boundary value problem (abbreviated to BVP in the sequel).

$$
A u=p(x, u, \nabla u)+f \text { in } G
$$

$$
u=0 \text { on } \partial G
$$

where $A$ is a nonlinear elliptic differential operator in divergence form of Leray-Lions type, $\nabla u=\operatorname{grad} u, f$ is a distribution on $G$ and $p(x, t, \eta)$ is a function defined on $G \times \mathbf{R} \times \mathbf{R}^{N}$. Among other hypotheses we shall, roughly speaking, assume that $p$ has completely unrelated growth rates in the first and the third variables. In this paper we prove the solvability of the BVP (1), (2) under the assumption that it has both an upper solution $\psi$ and a lower solution $\varphi$ with $\varphi \leqq \psi$.

Similar problems are considered, among others, by P. Hess in [3], [4] and the author in [1], [2]. In [3] the growth of the function $p$ is more restricted than allowed here and in [4] the domain is bounded. Our result seems to provide answer to a question raised in a remark at the end of [4] as to whether its result for bounded domains could be extended to unbounded ones. In [1] the conditions imposed on the upper and lower solutions $\varphi$ and $\psi$ are weaker than those assumed in this paper: namely, it is assumed in [1] that $\varphi$ and $\psi$ are local in character, i.e., they belong to some space of functions with some local properties. Then beside the fact that the solution obtained is also local in nature we had to essentially restrict ourselves to linear operators $A$ and it seems to us that the method of [1] cannot be adapted to nonlinear operators. While the main concern of [2] is solvability in weighted Sobolev's spaces using the result of [1] (thus the elliptic operators considered in [2] are linear), by specialization, i.e., by taking the weights equal to 1 , we have already obtained in [2] (cf. its Theorem 2) a weak version of Theorem 3 below for linear operators.

Received July 6, 1983 and in revised form May 2, 1984. 
The method of upper and lower solutions is conceptually simple and particularly useful in proving solvability of noncoercive BVPs. However, in practice it is severely limited by the difficulty encountered in constructing an upper solution $\psi$ and a lower solution $\varphi$ with $\varphi \leqq \psi$. This difficulty is genuinely nontrivial if the domain is unbounded and we want, as in some of our theorems further down, the upper and lower solutions to be simultaneously bounded and to belong to some space

$$
W_{\mathrm{loc}}^{1, q}(G) \cap L^{r}(G), \quad 1<q, r<\infty,
$$

and the operator is nonlinear. Thus for illustrative purposes we shall give an example for which one of our theorems is applicable. We shall explicitly construct upper (and lower) solutions by "gluing" together upper (and lower) solutions on subsets of $G$.

Finally we note that in order not to complicate the presentation we have not stated our results with their optimal hypotheses: for example, the theorems remain valid for more general unbounded domains than the exterior of a bounded one and the growth of $p(x, t, \eta)$ can be liberalized somewhat as in [4].

2. Notations, definitions and basic assumptions. Let

$$
A u=-D_{i}\left[A_{i}(x, u, \nabla u)\right], \quad D_{i}=\frac{\partial}{\partial x_{i}} .
$$

Here and in the sequel we use the convention that if the index $i$ is repeated then summation over that index from 1 to $N$ is implied. We make throughout the paper the following assumptions of Leray-Lions type concerning the functions $A_{i}, i=1, \ldots, N$ :

(H1) Each $A_{i}: G \times \mathbf{R} \times \mathbf{R}^{N} \rightarrow R$ is of Caratheodory's type, i.e., for each $(t, \eta) \in \mathbf{R} \times \mathbf{R}^{N}$ the function

$$
x \rightarrow A_{i}(x, t, \eta)
$$

is measurable and for almost all (a.a.) $x \in G$, the function

$$
(t, \eta) \rightarrow A_{i}(x, t, \eta)
$$

is continuous. Furthermore there exist constants $q, 1<q<\infty, c_{0} \geqq 0$ and a function

$$
k_{0}(\cdot) \in L_{\mathrm{loc}}^{q^{*}}(G) \quad\left(q^{*}=\frac{q}{q-1}\right), k_{0}(x) \geqq 0 \text { a.a. } \quad x \in G
$$

such that

$$
\left|A_{i}(x, t, \eta)\right| \leqq k_{0}(x)+c_{0}\left(|t|^{q-1}+|\eta|^{q-1}\right), \quad i=1, \ldots, N
$$

for a.a. $x \in G, \forall(t, \eta) \in \mathbf{R} \times \mathbf{R}^{N}$. Here and in the sequel, a function 
belongs to $L_{\text {loc }}^{q^{*}}(G)$ if its restriction to any bounded subset $E$ of $G$ belongs to $L^{q^{*}}(E)$.

(H2) $\left[A_{i}\left(x, t, \eta^{\prime}\right)-A_{i}(x, t, \eta)\right]\left(\eta_{i}^{\prime}-\eta_{i}\right)>0$

for a.a. $x \in G, \forall t \in \mathbf{R}$ if $\eta^{\prime} \neq \eta$ in $\mathbf{R}^{N}$.

(H3) $A_{i}(x, t, \eta) \eta_{i} \geqq \nu|\eta|^{q}$

for a.a. $x \in G, \forall(t, \eta) \in \mathbf{R} \times \mathbf{R}^{N}$ with some constant $\nu>0$.

Concerning the function $p(x, t, \eta)$ we assume throughout the paper:

(H4) The function $p(x, t, \eta)$ is of Caratheodory's type and there exists a constant $c_{1} \geqq 0$, a constant $\epsilon, 0<\epsilon \leqq q$, a function $k_{1}(\cdot) \in L_{\text {loc }}^{r}(G)$ for some $r \geqq 1, k_{1}(x) \geqq 0$ a.a. $x \in G$ such that

$$
|p(x, t, \eta)| \leqq k_{1}(x)+c_{1}|\eta|^{q-\epsilon}
$$

for a.a. $x \in G, \forall(t, \eta) \in \mathbf{R} \times \mathbf{R}^{N}$.

Let

$$
\begin{aligned}
& s=\min \left(r, \frac{q}{q-\epsilon}\right), \\
& s^{*}=\frac{s}{s-1} \text { if } s>1 \text { and } \\
& s^{*}=\infty \quad \text { if } s=1 .
\end{aligned}
$$

Definition 1. Suppose that $f \in W_{\mathrm{loc}}^{-1, q^{*}}\left(G_{n}\right)$. A function $\varphi \in W_{\mathrm{loc}}^{1, q}(G)$ is called a lower solution in the local sense of the BVP (1), (2) if

$$
\begin{aligned}
& \varphi \leqq 0 \quad \text { on } \partial G, \\
& p(x, \varphi, \nabla \varphi) \in L_{\mathrm{loc}}^{s}(G) \text { and } \\
& \int_{G} A_{i}\left(x, \varphi, \nabla_{\boldsymbol{\varphi}}\right) D_{i} v d x \leqq \int_{G} p(x, \varphi, \nabla \boldsymbol{\varphi}) v d x+\langle f, v\rangle
\end{aligned}
$$

for all $v \in W_{0}^{1, q}(G) \cap L^{s^{*}}(G)$ of compact support and $v \geqq 0$ a.e. (= almost everywhere) in $G$ where $\langle\cdot, \cdot\rangle$ denotes the pairing between $W_{0}^{1, q}(G)$ and its dual $W^{-1, q^{*}}(G)$.

An upper solution in the local sense is defined by reversing the inequality sign in the above definition.

Definition 2. Suppose that $f \in W_{\mathrm{loc}}^{-1, q^{*}}(G)$. A function $u \in W_{\mathrm{loc}}^{1, q}(G)$ is called a solution in the local sense of the BVP (1), (2) if $u=0$ on $\partial G$, $p(x, u, \nabla u) \in L_{\mathrm{loc}}^{s}(G)$ and

$$
\langle A u, v\rangle \stackrel{\text { Def }}{=} \int_{G} A_{i}(x, u, \nabla u) D_{i} v d x=\int_{G} p(x, u, \nabla u) v d x+\langle f, v\rangle
$$

for all $v \in W_{0}^{1, q}(G) \cap L^{s^{*}}(G)$ of compact support. If the subscripts "loc" 
are dropped and the equation is satisfied for all

$$
v \in W_{0}^{1, q}(G) \cap L^{r^{*}}(G) \cap L^{q / \epsilon}(G)
$$

then $u$ is called a solution.

3. The results. To prove our results we shall need the following

THEOREM 0 . Let $\Omega$ be a bounded subdomain of $G$ with smooth boundary $\partial \Omega$ and let $f \in W^{-1, q^{*}}(\Omega)$. Suppose that the BVP

$$
A u=p(x, u, \nabla u)+f \text { in } \Omega, \quad u=0 \text { on } \partial \Omega,
$$

has an upper solution $\psi$ and a lower solution $\varphi$ both belonging to $W^{1, q}(\Omega) \cap$ $L^{s^{*}}(\Omega)$ with $\varphi(x) \leqq \psi(x)$ for a.a. $x \in \Omega$. Then the BVP has a solution $u \in W_{0}^{1, q}(\Omega)$ with

$$
\varphi(x) \leqq u(x) \leqq \psi(x) \text { for a.a. } x \in \Omega .
$$

Proof. This theorem has been proved in [4] when in condition (H4) $r=1$ (thus $s=1, s^{*}=\infty$ ). With obvious modifications the proof of [4] still works for other values of $s \geqq 1$.

THEOREM 1. Let $f \in W^{-1, q^{*}}(G)$. Suppose that in $(\mathrm{H} 1)$ the function $k_{0}(\cdot) \in L^{q^{*}}(G)$ and in $(\mathrm{H} 4)$ the function $k_{1}(\cdot) \in L^{r^{*}}(G)$. Suppose also that the BVP (1), (2) has an upper solution $\psi$ and a lower solution $\varphi$ both in the local sense and both belonging to $L^{q}(G) \cap L^{r^{*}}(G) \cap L^{q / \epsilon}(G)$ with $\varphi(x) \leqq 0 \leqq \psi(x)$ for a.a. $x \in G$. Then that BVP has a solution $u \in W_{0}^{1, q}(G)$ with

$$
\varphi(x) \leqq u(x) \leqq \psi(x) \text { for a.a. } x \in G .
$$

Remark 1. For $r=q^{*}$ and $\epsilon=1$, Theorem 1 has been proved in [3] using a different method.

Remark 2. It is not difficult to see that for $0<\epsilon \leqq 1$, if $\varphi, \psi \in L^{q}(G) \cap$ $L^{\infty}(G)$ then $\varphi, \psi \in L^{q / \epsilon}(G)$ as well.

Remark 3. By performing a change of the unknown function as in [4], it can be seen that the theorem remains valid if we only have $\varphi(x) \leqq \psi(x)$ for a.a. $x \in G$.

Proof of Theorem 1. We suppose that $1 \leqq r<\infty$ and $0<\epsilon<q$. For other cases the proofs are similar. For each number $\rho>0$ let $B_{\rho}$ be the open ball in $\mathbf{R}^{N}$ with the center at the origin and with radius $\rho$ and let

$$
G_{\rho}=G \cap B_{\rho}
$$

We fix a number $n_{0}$ such that $\bar{D} \subset B_{n_{0}}$. For each integer $n>n_{0}$ consider the BVP

$$
A u=p(x, u, \nabla u)+f \text { in } G_{n}, \quad u=0 \text { on } \partial G_{n} .
$$


By Theorem 0 it has a solution $u_{n} \in W_{0}^{1, q}\left(G_{n}\right)$ with $\varphi \leqq u_{n} \leqq \psi$ a.e. on $G_{n}$ : Thus for each $v \in W_{0}^{1, q}\left(G_{n}\right) \cap L^{s^{*}}\left(G_{n}\right)$ we have

$$
\int_{G_{n}} A_{i}\left(x, u_{n}, \nabla u_{n}\right) D_{i} v d x=\int_{G_{n}} p\left(x, u_{n}, \nabla u_{n}\right) v d x+\langle f, v\rangle
$$

Taking $v=u_{n}$ we deduce from (H3) and (H4) that

$$
\nu \int_{G_{n}}\left|\nabla u_{n}\right|^{q} d x \leqq K_{1} \int_{G_{n}}\left\{k_{1}(x)\left|u_{n}\right|+\left|\nabla u_{n}\right|^{q-\epsilon}\left|u_{n}\right|\right\} d x+\left\langle f, u_{n}\right\rangle
$$

here and in the sequel $K_{j}(j=1,2, \ldots)$ denotes a constant $>0$, independent of $n$, not necessarily always the same. Writing

$$
f=h-D_{i} h_{i} \quad \text { with } h, h_{i} \in L^{q^{*}}(G), \quad i=1, \ldots, N
$$

and taking into account the fact that $\varphi \leqq u_{n} \leqq \psi$ a.e. on $G_{n}$ with $\varphi$, $\psi \in L^{q}(G)$ we obtain

$$
\left\langle f, u_{n}\right\rangle \leqq K_{2}+K_{3}\left(\int_{G_{n}}\left|\nabla u_{n}\right|^{q} d x\right)^{1 / q} .
$$

By Hölder's inequality:

$$
\begin{aligned}
\int_{G_{n}}\left|\nabla u_{n}\right|^{q-\epsilon}\left|u_{n}\right| d x & \leqq\left(\int_{G_{n}}\left|\nabla u_{n}\right|^{q} d x\right)^{1-\epsilon / q}\left(\int_{G_{n}}\left|u_{n}\right|^{\frac{q}{\epsilon}} d x\right)^{\epsilon / q} \\
& \leqq K_{4}\left(\int_{G_{n}}\left|\nabla u_{n}\right|^{q} d x\right)^{1-\epsilon / q}
\end{aligned}
$$

because $\varphi \leqq u_{n} \leqq \psi$ on $G_{n}$ and $\varphi, \psi \in L^{\frac{q}{\epsilon}}(G)$. Furthermore, because $k_{1}(\cdot) \in L^{r}(G)$ and $\varphi, \psi \in L^{r^{*}}(G)$, we deduce from (5), (6) and (7) that

$$
\left\|u_{n}\right\|_{W_{0}^{1, q}\left(G_{n}\right)} \leqq K_{5} \text {. }
$$

We extend $u_{n}$ to the whole domain $G$ by defining $u_{n}(x)=0$ when $x \in$ $G-G_{n}$ and for convenience, we still denote by $u_{n}$ the function so obtained. By the Sobolev imbedding theorem, and by using a diagonal process we deduce from (8) that we can extract from $\left\{u_{n}\right\}$ a subsequence which we still denote by $\left\{u_{n}\right\}$ such that

$$
\begin{aligned}
& \left\{u_{n}\right\} \text { converges weakly in } W_{0}^{1, q}(G) \text { to } u, \\
& \left\{u_{n}\right\} \text { converges almost everywhere on } G \text { to } u \text {. }
\end{aligned}
$$

Let $m$ be an arbitrary integer $>n_{0}$ and let $\zeta_{m}(\cdot)$ be a function in $C^{1}(G)$ with the following properties:

$$
\zeta_{m}(x) \in[0,1] \forall x \in G, \zeta_{m}(x)=1 \text { for } x \in G_{m}, \zeta_{m}(x)=0
$$

for $x \notin G_{m+1}$ and $\left|\nabla \zeta_{m}(\cdot)\right|$ is bounded on $G$ by a constant independent of $m$. 
In (4) with $n \geqq m+1$ we replace $v$ by $\zeta_{m}\left(u_{n}-u\right)$. We have

$$
\begin{aligned}
& \left|\int_{G_{n}} p\left(x, u_{n}, \nabla u_{n}\right) \zeta_{m}\left(u_{n}-u\right) d x\right| \\
& \leqq K_{1} \int_{G} k_{1}(x) \zeta_{m}\left|u_{n}-u\right| d x+K_{1} \int_{G}\left|\nabla u_{n}\right|^{q-\epsilon} \zeta_{m}\left|u_{n}-u\right| d x
\end{aligned}
$$

Since $k_{1}(x)\left|u_{n}-u\right| \rightarrow 0$ a.e. on $G$ as $n \rightarrow \infty$ and

$$
k_{1}(x)\left|u_{n}-u\right| \leqq 2[|\varphi|+|\psi|] k_{1}(x)
$$

by the Lebesgue dominated convergence theorem, the first integral on the right hand side of (9) tends to 0 as $n \rightarrow \infty$. Moreover $\left\{u_{n}\right\}$ converges strongly in $L^{q / \epsilon}(G)$ to $u$ because $\varphi \leqq u_{n}, u \leqq \psi ; \varphi, \psi \in L^{q / \epsilon}(G)$ and $\left\{u_{n}\right\}$ converges a.e. on $G$ to $u$; therefore the second integral on the right hand side of (9) also tends to 0 as $n \rightarrow \infty$. Thus

$$
\lim _{n \rightarrow \infty} \int_{G} p\left(x, u_{n}, \nabla u_{n}\right) \zeta_{m}\left(u_{n}-u\right) d x=0 .
$$

Since $\left\{u_{n}\right\}$ converges weakly in $W_{0}^{1, q}(G)$ to $u$ it is clear that

$$
\lim _{n \rightarrow \infty}\left\langle f, \zeta_{m}\left(u_{n}-u\right)\right\rangle=0 .
$$

Hence we deduce from (4) that

$$
\begin{aligned}
& \lim _{n \rightarrow \infty} \int_{G} A_{i}\left(x, u_{n}, \nabla u_{n}\right) D_{i}\left[\zeta_{m}\left(u_{n}-u\right)\right] d x \\
& =\lim _{n \rightarrow \infty} \int_{G} A_{i}\left(x, u_{n}, \nabla u_{n}\right) D_{i} \zeta_{m}\left(u_{n}-u\right) d x \\
& +\lim _{n \rightarrow \infty} \int_{G} A_{i}\left(x, u_{n}, \nabla u_{n}\right) \zeta_{m} D_{i}\left(u_{n}-u\right) d x \\
& =0 .
\end{aligned}
$$

In the last equation the first limit on its left hand side is 0 because the sequences $\left\{A_{i}\left(x, u_{n}, \nabla u_{n}\right)\right\}(i=1, \ldots, N)$ are bounded in $L^{q^{*}}(G)$ whereas $\left\{u_{n}\right\}$ converges strongly in $L^{q}\left(G_{m+1}\right)$ to $u$. Thus the second limit on the left hand side of the last equation is also 0 :

(10) $\lim _{n \rightarrow \infty} \int_{G} A_{i}\left(x, u_{n}, \nabla u_{n}\right) \zeta_{m} D_{i}\left(u_{n}-u\right) d x=0$.

Since $\left\{u_{n}\right\}$ converges strongly to $u$ in $L^{q}\left(G_{m+1}\right)$ we know (cf., e.g., [5], Lemma 2.1, page 183) that for each $i=1, \ldots, N,\left\{A_{i}\left(x, u_{n}, \nabla u\right)\right\}$ 
converges strongly to $A_{i}(x, u, \nabla u)$ in $L^{q^{*}}\left(G_{m+1}\right)$; because $\left\{u_{n}\right\}$ converges weakly in $W_{0}^{1, q}(G)$ to $u$ we therefore deduce that

$$
\lim _{n \rightarrow \infty} \int_{G} A_{i}\left(x, u_{n}, \nabla u\right) \zeta_{m} D_{i}\left(u_{n}-u\right) d x=0 .
$$

It follows from (10) and (11) that

$$
\lim _{n \rightarrow \infty} \int_{G}\left[A_{i}\left(x, u_{n}, \nabla u_{n}\right)-A_{i}\left(x, u_{n}, \nabla u\right)\right] \zeta_{m} D_{i}\left(u_{n}-u\right) d x=0
$$

and hence

$$
\lim _{n \rightarrow \infty} \int_{G_{m}}\left[A_{i}\left(x, u_{n}, \nabla u_{n}\right)-A_{i}\left(x, u_{n}, \nabla u\right)\right] D_{i}\left(u_{n}-u\right) d x=0 .
$$

We deduce from this (cf., e.g., [5], Proof of Lemma 2.2, page 184) that we can extract from $\left\{u_{n}\right\}$ a subsequence, which we still denote by $\left\{u_{n}\right\}$, such that $\left\{\nabla u_{n}\right\}$ converges a.e. to $\nabla u$ on $G_{m}$. Since this is true for any integer $m>n_{0}$, using a diagonal process, we see that we can extract from $\left\{u_{n}\right\}$ a subsequence, which we still denote by $\left\{u_{n}\right\}$, such that

$\left\{\nabla u_{n}\right\}$ converges a.e. to $\nabla u$ on $G$.

Then, for each $i=1, \ldots, N,\left\{A_{i}\left(x, u_{n}, \nabla u_{n}\right)\right\}$ converges a.e. to $A_{i}(x, u$, $\nabla u)$ on $G$; because those sequences are also bounded in $L^{q^{*}}(G)$, we conclude that

$$
\left\{A_{i}\left(x, u_{n}, \nabla u_{n}\right)\right\} \text { converges weakly to } A_{i}(x, u, \nabla u) \text { in } L^{q^{*}}(G) .
$$

We now show that for every $w \in L^{q / \epsilon}(G) \cap L^{r^{*}}(G) \cap W_{0}^{1, q}(G)$ we have

$$
\lim _{n \rightarrow \infty} \int_{G} p\left(x, u_{n}, \nabla u_{n}\right) w d x=\int_{G} p(x, u, \nabla u) w d x .
$$

Let $\epsilon^{\prime}>0$ be arbitrarily given. We first choose and fix an integer $m$ sufficiently large, $m>n_{0}$, so that we have simultaneously

(12) $\left(\int_{G-G_{m}}\left[k_{1}(x)\right]^{r} d x\right)^{\frac{1}{r}}<\epsilon^{\prime}$ and $\left(\int_{G-G_{m}}|w|^{\frac{q}{\epsilon}} d x\right)^{\frac{\epsilon}{q}}<\epsilon^{\prime}$.

We next choose $\delta>0$ such that if $E \subset G_{m}$, $\operatorname{mes}(E)<\delta$, then we have simultaneously

$$
\left.\left(\int_{E}\left[k_{1}(x)\right]^{r} d x\right)^{\frac{1}{r}}<\epsilon^{\prime} \text { and }\left[\int_{E}|w|^{\frac{q}{\epsilon}} d x\right)\right]^{\frac{\epsilon}{q}}<\epsilon^{\prime} .
$$

(This can be done by approximating $k_{1}(\cdot)$ in $L^{r}(G)$ and $w(\cdot)$ in $L^{q / \epsilon}(G)$ by step functions first.) By Egoroff's theorem we can find a subset $E_{0}$ of $G_{m}$ with mes $\left(E_{0}\right)<\delta$ such that on $G_{m}-E_{0}$ the sequence $\left\{p\left(x, u_{n}, \nabla u_{n}\right)\right\}$ 
converges uniformly to $p(x, u, \nabla u)$ because $\left\{p\left(x, u_{n}, \nabla u_{n}\right)\right\}$ converges a.e. on $G$ to $p(x, u, \nabla u)$. Since $w \in L^{r *}(G)$ and $\operatorname{mes}\left(G_{m}\right)<\infty$, an integer $L$ can be found such that

$$
\left|\int_{G_{m}-E_{0}}\left[p\left(x, u_{n}, \nabla u_{n}\right)-p(x, u, \nabla u)\right] w(x) d x\right|<\epsilon^{\prime} \quad \text { if } n>L .
$$

By hypothesis (H4) we have

$$
\begin{aligned}
& \left|\int_{E_{0}}\left[p\left(x, u_{n}, \nabla u_{n}\right)-p(x, u, \nabla u)\right] w(x) d x\right| \\
& <2 \int_{E_{0}} k_{1}(x)|w(x)| d x \\
& +c_{1} \int_{E_{0}}\left(\left|\nabla u_{n}\right|^{q-\epsilon}+|\nabla u|^{q-\epsilon}\right)|w(x)| d x \\
& <2 \epsilon^{\prime}\|w\|_{L^{*}(G)}+2 c_{1} \epsilon^{\prime} K_{5}^{q-\epsilon}
\end{aligned}
$$

by (13) where $K_{5}$ is the constant in (8). Similarly,

$$
\begin{aligned}
& \left|\int_{G-G_{m}}\left[p\left(x, u_{n}, \nabla u_{n}\right)-p(x, u, \nabla u)\right] w d x\right| \\
& <2 \epsilon^{\prime}\left(\|w\|_{L^{*}(G)}+K_{6}^{q-\epsilon}\right)
\end{aligned}
$$

by (12). Because $\epsilon^{\prime}>0$ is arbitrary, we deduce from the last three inequalities that for every $w \in L^{q / \epsilon}(G) \cap L^{r^{*}}(G) \cap W_{0}^{1, q}(G)$,

$$
\lim _{n \rightarrow \infty} \int_{G} p\left(x, u_{n}, \nabla u_{n}\right) w d x=\int_{G} p(x, u, \nabla u) w d x .
$$

Given such a $w$, taking $v=\zeta_{m} w$ in (4) with $n \geqq m+1$ we have by letting $n \rightarrow \infty$,

$$
\left\langle A u, \zeta_{m} w\right\rangle=\int_{G} p(x, u, \nabla u) \zeta_{m} w d x+\left\langle f, \zeta_{m} w\right\rangle
$$

Letting $m \rightarrow \infty$ we conclude that $u$ is a solution of the BVP (1), (2).

We now give a few possible variations of Theorem 1 .

If we relax the requirements of the operator $A$ then we can only prove the existence of a solution in the local sense.

We recall we always assume that conditions $(\mathrm{H} 1)-(\mathrm{H} 4)$ of Section 2 are satisfied.

THeOREM 2. Let $f \in W^{-1, q^{*}}(G)$. Suppose further that in (H4) the function

$$
k_{1}(\cdot) \in L^{r}(G) \cup L^{q^{*}}(G) \cup L^{q /(q-\epsilon)}(G) .
$$

Suppose also that the BVP (1), (2) has an upper solution $\psi$ and a lower solution $\varphi$ both in the local sense and both belonging to $L^{r^{*}}(G) \cap L^{q / \epsilon}(G) \cap$ 
$L^{q}(G)$ with $\varphi(x) \leqq 0 \leqq \psi(x)$ for a.a. $x \in G$. Then that BVP has a solution in the local sense $u \in W_{0}^{1, q}(G)$ with

$$
\varphi(x) \leqq u(x) \leqq \psi(x) \text { for a.a. } x \in G .
$$

Proof. The proof is similar to the proof of Theorem 1. The only difference is that now we can no longer prove that $\left\{A_{i}\left(x, u_{n}, \nabla u_{n}\right)\right\}$ converges to $A_{i}(x, u, \nabla u)(i=1, \ldots, N)$ in $L^{q^{*}}(G)$. We can only prove that the sequence of restrictions $\left\{\left.A_{i}\left(x, u_{n}, \nabla u_{n}\right)\right|_{G_{m}}\right\}$ converges to the restriction $\left.A_{i}(x, u, \nabla u)\right|_{G_{m}}$ for any $m>n_{0}$ because in condition $(\mathrm{H1})$ it is merely assumed that

$$
k_{0}(\cdot) \in L_{\text {loc }}^{q^{*}}(G) \text {. }
$$

We also have the following special case of Theorem 2 which generalizes Theorem 2 of [2] to nonlinear operators:

THEOREM 3. Suppose that in (H4) the function

$$
k_{1}(\cdot) \in L^{r}(G) \cup L^{q /(q-\epsilon)}(G) .
$$

Suppose also that the BVP

$$
\begin{aligned}
& A u=p(x, u, \nabla u)-D_{i} h_{i} \text { on } G \\
& u=0 \text { on } \partial G
\end{aligned}
$$

with $h_{i} \in L^{q^{*}}(G)$ has an upper solution $\psi$ and a lower solution $\varphi$ both in the local sense and both belonging to

$$
W_{\mathrm{loc}}^{1, q}(G) \cap L^{r^{*}}(G) \cap L^{q / \epsilon}(G)
$$

with $\varphi(x) \leqq 0 \leqq \psi(x)$ for a.a. $x \in G$. Then it has a solution in the local sense $u \in W_{0}^{1, q}(G)$ with

$$
\varphi(x) \leqq u(x) \leqq \psi(x) \text { for a.a. } x \in G .
$$

Proof. The proof is similar to the proof of Theorem 2. In Theorem 2 we require in addition that $\varphi, \psi \in L^{q}(G)$ only to insure that with

$$
f=h-D_{i} h_{i} \in W^{-1, q^{*}}(G)
$$

we have (with $u_{n}$ defined by (4))

$$
\left|\int_{G_{n}} h u_{n} d x\right|<K_{7} \quad \forall n>n_{0} .
$$

But in Theorem $2, h=0$ and that additional requirement is no longer necessary.

For the proof of Theorem 5 we shall need the following generalized version of Theorem 2 . We consider a function $d(\cdot): \mathbf{R} \rightarrow \mathbf{R}$ satisfying the following hypothesis:

(H5) $d(\cdot)$ is continuous on $\mathbf{R}$ and $d(t) t \geqq 0 \forall t \in \mathbf{R}$. 
THEOREM 4. Suppose that $f \in W^{-1, q^{*}}(G)$ and in $(\mathrm{H} 4)$ the function

$$
k_{1}(\cdot) \in L^{1}(G) \cup L^{q^{*}}(G) \cup L^{q /(q-\epsilon)}(G) .
$$

Suppose also that the BVP

$$
\begin{aligned}
& A u+d(u)=p(x, u, \nabla u)+f \text { on } G \\
& u=0 \text { on } \partial G
\end{aligned}
$$

has an upper solution $\psi$ and a lower solution $\varphi$ both in the local sense and both belonging to

$$
W_{\mathrm{loc}}^{1, q}(G) \cap L^{\infty}(G) \cap L^{q}(G) \cap L^{q / \epsilon}(G)
$$

with $\varphi(x) \leqq 0 \leqq \psi(x)$ for a.a. $x \in G$. Then it has a solution in the local sense $u \in W_{0}^{1, q}(G)$ with

$$
\varphi(x) \leqq u(x) \leqq \psi(x) \text { for a.a. } x \in G .
$$

Proof. The proof is similar to the proof of Theorem 1 taking into account the difference already noted in the proof of Theorem 2. Now in (4) there is an additional term $\int_{G_{n}} d\left(u_{n}\right) v d x$. When we take $v=u_{n}$ to arrive at (8) this term drops out because

$$
d(t) t \geqq 0 \forall t \in \mathbf{R} .
$$

Furthermore, with $\varphi \leqq u_{n} \leqq \psi \quad \forall n=n_{0}, n_{0}+1, \ldots$ and $u_{n} \rightarrow u$ a.e. on $G$ as $n \rightarrow \infty$ as in the proof of Theorem 1, for every integer $m>n_{0}$ we have

$$
\lim _{n \rightarrow \infty} \int_{G} d\left(u_{n}\right) \zeta_{m}\left(u_{n}-u\right) d x=0
$$

because the sequence $\left\{d\left(u_{n}\right)\right\}$ is bounded in $L^{\infty}\left(G_{m+1}\right)$ and $\left\{u_{n}\right\}$ converges strongly to $u$ in $L^{q}\left(G_{m+1}\right)$. Hence (10) is still valid.

Remark 4. Suppose that, in Theorem 5,

$$
f=0 \text { and } k_{1}(\cdot) \in L^{q / q-\epsilon}(G) .
$$

(i) Then it is not difficult to see that it suffices to require

$$
\boldsymbol{\varphi}, \psi \in W_{\mathrm{loc}}^{1, q}(G) \cap L_{\mathrm{loc}}^{\infty}(G) \cap L^{q / \epsilon}(G)
$$

for the theorem to hold.

(ii) Even if the operator $A$ satisfies the stronger assumption of Theorem 1 that in $(\mathrm{H} 1) k_{0}(\cdot) \in L^{q^{*}}(G)$ we can still prove only that $u$ is a solution in the local sense of the BVP because in general we cannot prove that for every $v \in W_{0}^{1, q}(G) \cap L^{q / \epsilon}(G)$ we have

$$
\lim _{n \rightarrow \infty} \int_{G} d\left(u_{n}\right) v d x=\int_{G} d(u) v d x
$$


For illustrative purposes we now give a specific BVP for which we can show the existence of a solution by applying Theorem 4. This BVP is adapted from the one in [2].

THEOREM 5. Suppose that $d: \mathbf{R} \rightarrow \mathbf{R}$ is differentiable, $d(0)=0, d^{\prime}(t) \geqq \lambda$ $>0 \forall t \in \mathbf{R}$;

$$
|p(x, t, \eta)| \leqq k(x)+c(\rho)|\eta|^{q-\epsilon} \quad \forall|t|<\rho
$$

where $2 \leqq q<\infty, 0<\epsilon \leqq q-1, k(x) \geqq 0$ for a.a. $x \in G$ and $k(\cdot) \in L^{\infty}(G), k(x)|x|^{\alpha} \rightarrow 0$ as $|x| \rightarrow \infty$ uniformly with

$$
\alpha>\max \left(\frac{N \epsilon}{q}, \frac{N(q-\epsilon)}{q}\right) .
$$

Then the BVP

(15) $u=0$ on $\partial G$

has a solution in the local sense $u \in W_{0}^{1, q}(G)$.

Proof. Since $k(\cdot) \in L^{q / q-\epsilon}(G)$, by Theorem 4 and Remark 4 (i) following it, it suffices to construct an upper solution $\psi$ and a lower solution $\varphi$, with $\varphi \leqq 0 \leqq \psi$ on $G ; \varphi, \psi \in L^{\infty}(G) \cap L^{q / \epsilon}(G)$, of the BVP (14), (15). For that purpose we first fix a number $M>1$ such that

(16) $\lambda M>\|k(\cdot)\|_{L^{\infty}(G)}$.

For $x \neq 0$ let $\psi_{1}(x)=M \rho^{\alpha}|x|^{-\alpha}, \rho>0$ to be determined. Using the fact that $0<\epsilon \leqq q-1$ and $d(t) \geqq \lambda t \forall t>0$ it can be shown by direct computation that we can choose a number $\rho$ large enough so that for $|x|>\rho$ we have

$$
-D_{i}\left[\left|D_{i} \psi_{1}\right|^{q-2} D_{i} \psi_{1}\right]+d\left(\psi_{1}\right) \geqq p\left(x, \psi_{1}, \nabla \psi_{1}\right) .
$$

We set

$$
\psi(x)=\psi_{1}(x) \text { if }|x| \geqq \rho, \quad \psi(x)=M \text { if }|x|<\rho .
$$

We shall show that $\psi$ is an upper solution of the BVP in the sense of Definition 1 . We note that clearly $\psi>0$ on $G$ and

$$
\psi(\cdot) \in L^{\infty}(G) \cap L^{q / \epsilon}(G) \cap W_{\mathrm{loc}}^{1, q}(G) .
$$

It suffices to show that for any $v \in C_{0}^{\infty}(G)$ with $v \geqq 0$ on $G$ we have

$$
\int_{G}\left|D_{i} \psi\right|^{q-2} D_{i} \psi D_{i} v d x+\int_{G} d(\psi) v d x \geqq \int_{G} p(x, \psi, \nabla \psi) v d x
$$

In fact, because

$$
D_{i} \psi_{1} \cdot \frac{x_{i}}{\rho}<0
$$


integration by parts gives

$$
\begin{aligned}
\int_{|x| \geqq \rho}-D_{i}\left[\left|D_{i} \psi\right|^{q-2} D_{i} \psi\right] v d x & =\int_{|x|=\rho}\left|D_{i} \psi\right|^{q-2} D_{i} \psi v \frac{x_{i}}{\rho} d x \\
& +\int_{|x| \geqq \rho}\left|D_{i} \psi\right|^{q-2} D_{i} \psi D_{i} v d x \\
& \leqq \int_{|x| \geqq \rho}\left|D_{i} \psi\right|^{q-2} D_{i} \psi D_{i} v d x
\end{aligned}
$$

Therefore, by (17),

$$
\begin{aligned}
& \int_{|x| \geqq \rho}\left|D_{i} \psi\right|^{q-2} D_{i} \psi D_{i} v d x+\int_{|x| \geqq \rho} d(\psi) v d x \\
& \geqq \int_{|x| \geqq \rho} p(x, \psi, \nabla \psi) v d x .
\end{aligned}
$$

Furthermore, with $G_{\rho}=G \cap B_{\rho}$, it follows from (16) that

$$
\int_{G_{\rho}}\left|D_{i} \psi\right|^{q-2} D_{i} \psi D_{i} v d x+\int_{G_{\rho}} d(\psi) v d x \geqq \int_{G_{\rho}} p(x, \psi, \nabla \psi) v d x
$$

(18) then follows from (19) and (20). A negative lower solution

$$
\varphi \in L^{\infty}(G) \cap L^{q / \epsilon}(G) \cap W_{\mathrm{loc}}^{\mathrm{l}, q}(G)
$$

is constructed similarly.

The author is indebted to an anonymous referee for constructive observations.

\section{REFERENCES}

1. N. P. Các, Nonlinear elliptic boundary value problems for unbounded domains, J. Differential Equations 45 (1982), 191-198.

2. On some quasilinear elliptic boundary value problems with conditions at infinity, J. Differential Equations 52 (1984), 342-355.

3. P. Hess, Nonlinear elliptic problems in unbounded domains, International Summer School on Nonlinear Operators, Berlin, Sept. 1975, in "Abhandlung der Akademie der Wissenschafter der DDR."

4. - A second order nonlinear elliptic boundary value problem, in "Nonlinear Analysis: A collection of papers in honor of Erich H. Rothe," (Academic Press, New York, 1978).

5. J. L. Lions, Quelques méthodes de résolution des problèmes aux limites nonlinéaires (Dunod, Gauthier-Villars, Paris, 1969).

The University of Iowa,

Iowa City, Iowa 